\title{
PlasticNet: Deep learning for automatic microplastic recognition via FT-IR spectroscopy
}

Ziang Zhu

Wayne Parker

Alexander Wong

Email: $\{z 259 z h u$,
Department of Civil and Environmental Engineering, University of Waterloo

Department of Civil and Environmental Engineering, University of Waterloo

Department of Systems Design Engineering \& Waterloo Artificial Intelligence Institute, University of Waterloo

\section{Abstract}

The recognition of microplastics (MPs) in environmental samples via Fourier transform infrared spectroscopy (FT-IR) is challenging due to a plethora of factors such as the presence of plasticizers, weathering, and contamination that can lead to significant variances in measured spectra for a given type of plastic. Conventional library search approaches proposed in literature rely on comparing the observed spectrum with spectra in reference libraries, and thus significant spectra variances can lead to recognition errors when leveraging such approaches. Motivated to tackle this challenge, this study explores the feasibility of leveraging deep learning for automatic MP recognition via FT-IR spectroscopy. More specifically, a deep convolution neural network (CNN) architecture, referred to here as PlasticNet, is introduced for the purpose of automatic MP recognition. PlasticNet was trained on a large corpus of FT-IR spectra of different plastic types in order to learn discriminative spectral features characterizing each plastic type. Experimental results showed that PlasticNet was capable of recognizing between polypropylene (PP) and polyethelene (PE) particles from a sample in an effective and efficient manner, with PlasticNet correctly identifying PP particles that were not recognized via a library search technique and at a faster recognition speed as well.

\section{Introduction}

The ubiquitous presence of microplastics (MPs) and their potential threats to aquatic and human health have become a growing public concern [1] (Ziccardi et al., 2016). Common methods for MP analysis include visual sorting and single-point Fourier transform infrared spectroscopy (FT-IR) analysis. Visual sorting with the naked eye or dissecting microscopy is easy in operation but requires significant human labor and has been shown to have up to $70 \%$ error. Single-point FT-IR which analyzes MPs one by one is time consuming especially when there are a large number of MPs in the sample. The use of focal plane array based (FPA-based) micro-Fourier transform infrared spectroscopy (micro-FT-IR) is a promising technology for rapid and accurate identification of MPs in environmental samples because it simultaneously collects multiple spectra of MPs on a surface. The output of an FPA-based micro-FT-IR measurement is a 3-D matrix, with each pixel represented by $\mathrm{x}-\mathrm{y}$ coordinates and the associated FT-IR spectrum. A key question in data analysis is the composition of each pixel and by extension the MP particle.

The conventional approach for MP recognition is known as a library search strategy, where the correlation coefficient between an unknown spectrum and one or few standard spectra in a reference library is calculated to determine which plastic type has the closest matching reference spectrum to the unknown spectrum. However, weathering, heterogeneity of particles, and attachment of biofilms can significantly modify spectra of plastics [2] (Primpke et al., 2017) and thus lead to significant spectra variances for a given plastic type. As such, leveraging standard library search strategies can result in recognition errors due to low correlation between a given reference spectra and an unknown spectra under the aforementioned conditions. Therefore, the result in leveraging such library search methods in real-world scenarios could be the missed identification of MP particles.

Given the recent advances in the field of deep learning, there is a growing interest in leveraging deep convolution neural networks (CNN) for the purpose of automatic MP recognition. Rather than comparing with a limited set of reference spectra as with library search methods, CNNs are capable of directly learning discriminative spectral features characterizing each plastic type in a way that accounts for inherent spectral variances. For example, $\mathrm{Ng}$ et al., (2020) [3] recently leveraged a CNN for MP recognition on the basis of hyperspectral data. However, to the best of the authors' knowledge, the use of CNNs for automatic MP recognition via FT-IR spectroscopy has not been previously explored.

Motivated by the aforementioned potential, this study explores the feasibility of leveraging deep learning for automatic MP recognition via FT-IR spectroscopy. This is accomplished through the introduction of PlasticNet, a CNN architecture designed specifically for the purpose of automatic MP recognition based on FT-IR spectra. The methodology behind this study along with the corresponding experimental results will be discussed in the following sections.

\section{Methodology}

To expore the feasibility of leveraging deep learning for automatic MP recognition via FT-IR spectroscopy, we construct a tailored CNN architecture called PlasticNet. The PlasticNet network architecture is based on a VGG deep convolutional neural network architecture [4] (Simonyan \& Zisserman, 2014), and comprises of 8 convolution layers, 3 max pooling layers, 3 fully connected layers, and 1 softmax layer that outputs the likelihood of an input FT-IR spectrum belonging to each plastic type under consideration. Each 1-D FTIR spectrum is transformed to a 2-D spectrogram representation by sliding a Hamming window across the 1D spectrum and producing a 2-D stack of overlapping spectral segments. A Fourier transform is then applied to the spectral segment stack to obtain the final 2D spectrogram. By transforming the input FT-IR spectra into a 2-D spectrogram representation, one can better structure the spectral relationships between adjacent spectral frequencies in a way that facilitates for improved spectral feature learning by a CNN architecture such as PlasticNet.

Training PlasticNet requires a large number of spectra (a minimum of 200 for each type for optimal results) of each type of plastic type. The dataset used in this study for training and testing PlasticNet was obtained from [5] (Primpke et al., 2018). The samples from the dataset had been collected at a wastewater treatment plant, and was imaged by a Bruker Hyperion 3000 FPA-based FT-IR microscope after cleaning with enzymatic digestion. The extraction of data was performed using Bruker OPUS(C)7.5. A total of 415, 256 , and 921 spectra were obtained from the PP and PE MPs and the background respectively for training and testing PlasticNet. For each type of plastic, $50 \%$ of the samples were used for training PlasticNet and the other $50 \%$ were used for testing PlasticNet. The proposed PlasticNet was constructed, trained, and tested in Mathwork Matlab(C)R2018a. The flow chart of methodology is summarized in Fig.1.

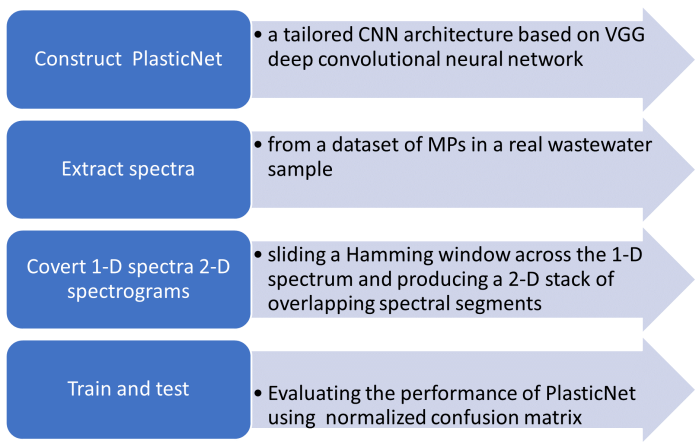

Fig. 1: Flow diagram of methodology

\section{Results and discussion}

The goal of this study was to critically assess the potential of deep learning for improved MP recognition via FT-IR spectroscopy when compared with library search techniques. The library search method that was used in this study for comparison was that described by [6] (Primpke et al., 2020). The following sections discuss the assessment of the accuracy of recognition by PlasticNet, present a further validation of the efficacy of PlasticNet by manually re-analyzing the spectra of recognized particles, and compare 



Fig. 2: Visualization of dataset used for training/testing the proposed PlasticNet. Particles 1 and 2 provided spectra for PP (yellow) and PE (red). Image (A) shows all particles recognized by the library search method while Image (B) shows particles recognized by PlasticNet after training. Particle 3 is an example of a PP MP that was recognized by PlasticNet but not by library search.

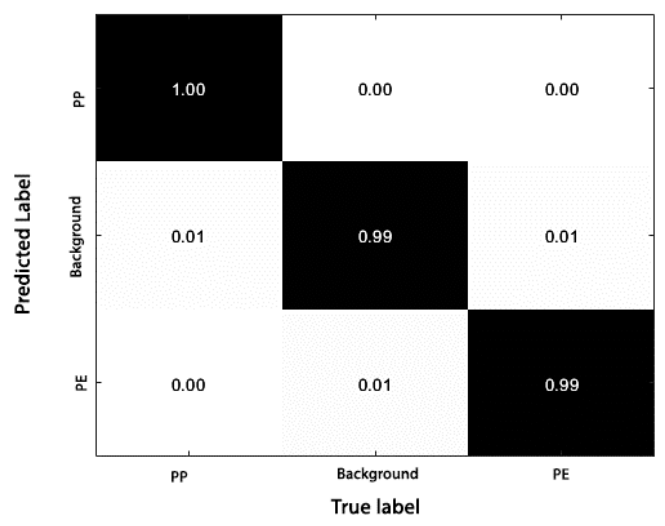

Fig. 3: Normalized Confusion Matrix.

the recognition speed achieved by PlasticNet when compared to a library search method. The latter was deemed important as excessive sample processing times will diminish the attractiveness of a method in practice.

As shown in Fig. 2A, several large PE (labelled as red) and PP (labelled as dark yellow) MPs were present in the dataset. The large particles allowed for the extraction of hundreds of spectra and hence all of the PP and PE spectra were extracted from particle 1 and particle 2 respectively. The spectrum at the particle edges were weaker and noisier compared with those in the center of the particles, so special attention was paid to extract spectra on the edge of the particles while making sure essential bands existed.

The automatic MP recognition performance of PlasticNet was visualized using a normalized confusion matrix and is shown in Fig.3. The diagonal elements of the confusion matrix represent the percentage of spectra for which the prediction was correct, while off-diagonal elements represent the percentage of spectra that were mispredicted by PlasticNet. As shown in Fig.3, the true positive percentage of PP, background, and PE were 1, 0.99, and 0.99 respectively, demonstrating a high recognition accuracy of the proposed PlasticNet.

To further explore the efficiacy of the proposed PlasticNet to recognize MPs, the recognition performance of PlasticNet was compared with that of a library search method. This involved examining MPs that were outside of the training sets, and focused on MPs that were recognized by PlasticNet but were not reported by the library search. Manual reanalysis, which examined the presence and absence of essential or additional bands of MPs, was performed for validation. As shown in Fig.4, particle 3 from Fig.2B, that appeared in PlasticNet's prediction and was not conclusively recognized by the library search method, is presented as an example of how the manual reanalysis was performed. As shown in Fig. 4C, all of the essential peaks of PP existed in the particle 3 spectra, demonstrating that it was composed of PP. A similar reanalysis was performed for 22 PP particles that were recognized by PlasticNet and missed by the library search method, and the essential PP peaks were found in all of them. Manual reanalysis was not done on the "additional" PE particles because the spectra of PE and acrylates are similar. This issue may be resolved when more acrylates spectra are added to PlasticNet training process. Overall, the analysis revealed that the proposed PlasticNet has the capability to automatically recognize MPs that cannot be recognized via library search.

Recognition speed is an important aspect of data analysis as the dataset derived from FPA FT-IR analysis of a environmental sample usually contains approximately one million spectra. The data analysis was performed on a desktop with an Intel E5 2687W processor, 64GB DDR3 memory, NVIDA GeForce RTX 2060 Super graphic card. Approximately $114 \mathrm{~min}$ was required for the library search to process the dataset while it took only 62 min for PlasticNet to process the same dataset. Hence, the proposed PlasticNet was $46 \%$ faster than the library search while producing higher quality identifications. This substantial reduction in time for analysis would reduce the resources required for intensive MP research. 


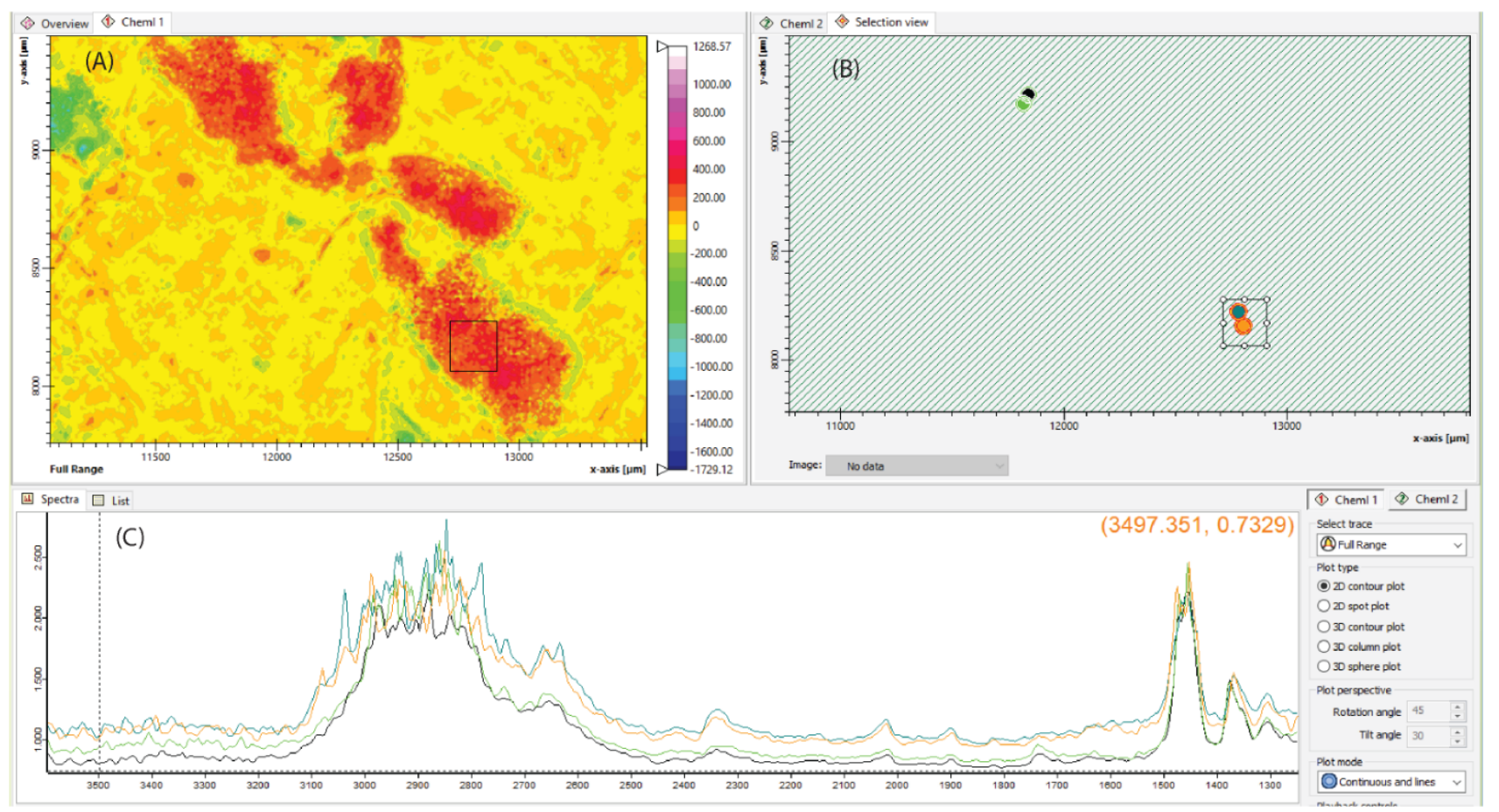

Fig. 4: Manual reanalysis of particle 3 that was recognized by PlasticNet but not by the library search method. (A) Particle images established by integrating alkane peaks. (B) Location of spectra selected for reanalysis. Dark green and orange circles were on particle 3 , while the black and light green circles were on a PP particle that was recognized by both PlasticNet and library search. (C) Spectra indicating essential PP peaks existed in particle 3.

\section{Conclusion}

This study explored the feasibility of leveraging deep learning for automatic MP recognition via FT-IR spectroscopy. This was facilitated by the introduction of PlasticNet, a deep convolutional neural network architecture tailored for MP recognition. Experimental results using plastic samples showed that the proposed PlasticNet can achieve not only improved MP recognition performance when compard to library search methods, but also perform MP recognition at a significant faster speed. Future work involves increasing the FT-IR spectra corpus in terms of both quantity of samples per plastic type but also the variety of plastic types, and exploring the performance of PlasticNet when trained on this expanded wealth of spectral data.

\section{References}

[1] L. M. Ziccardi, A. Edgington, K. Hentz, K. J. Kulacki, and S. K. Driscoll, "Microplastics as vectors for bioaccumulation of hydrophobic organic chemicals in the marine environment: A state-of-the-science review," Environmental toxicology and chemistry, vol. 35, no. 7, pp. 1667-1676, 2016.

[2] S. Primpke, C. Lorenz, R. Rascher-Friesenhausen, and G. Gerdts, "An automated approach for microplastics analysis using focal plane array (fpa) ftir microscopy and image analysis," Analytical Methods, vol. 9, no. 9, pp. 1499-1511, 2017.

[3] W. Ng, B. Minasny, M. Montazerolghaem, J. Padarian, R. Ferguson, S. Bailey, and A. B. McBratney, "Convolutional neural network for simultaneous prediction of several soil properties using visible/near-infrared, mid-infrared, and their combined spectra," Geoderma, vol. 352, pp. 251 - 267, 2019.

[4] K. Simonyan and A. Zisserman, "Very deep convolutional networks for large-scale image recognition," arXiv preprint arXiv:1409.1556, 2014.

[5] S. Primpke, M. Wirth, C. Lorenz, and G. Gerdts, "Reference database design for the automated analysis of microplastic samples based on fourier transform infrared (ftir) spectroscopy," Analytical and bioanalytical chemistry, vol. 410, no. 21, pp. 5131-5141, 2018.

[6] S. Primpke, R. K. Cross, S. M. Mintenig, M. Simon, A. Vianello, G. Gerdts, and J. Vollertsen, "Express: Toward the systematic identification of microplastics in the environment: Evaluation of a new independent software tool (simple) for spectroscopic analysis," Applied Spectroscopy, vol. 74, no. 9, pp. 1127-1138, 2020. 